\title{
Integrated Railway Infrastructure Management System with Uniform Location on a Kilometerage Basis
}

\author{
Takafumi YOSHIDA \\ Hirofumi TANAKA \\ Masahito NISHIMOTO \\ Track Geometry \& Maintenance Laboratory, Track Technology Division \\ Masashi MIWA \\ Track Technology Division
}

\begin{abstract}
For railway infrastructure management, railway companies carry out inspections and manage inspection data with ledger data, by 'field', such as track, civil engineering, electric power, and signal. If we can browse and analyze these data comprehensively, the quality of infrastructure management can be improved, eventually increasing running safety. Therefore, we are developing a system that integrates inspection data and ledger data from each field. In this paper, we show elemental technologies required for developing the system, after organizing merits of integrated data management. In addition, we show some examples of using the integrated management system for infrastructure management across fields.
\end{abstract}

Key words: position information, track maintenance, kilometerage, integrated management, railway infrastructure, infrastructure maintenance

\section{Introduction}

For railway infrastructure management, and for running safety, railway companies carry out inspections by 'field,' such as track, civil engineering, electric power, and signal. The inspection data is recorded and managed together with ledger data for each field. So far, these data are generally managed separately for each field and each inspection. In order to use the data from each field for maintenance, it is mainly analyzed to estimate the cause of deterioration of infrastructure. On the other hand, in recent years, infrastructure diagnosis and management methods that utilize data acquired from other fields have been proposed. For example, there is a method for detecting bridge resonance using data that is regularly measured onboard by inspection cars for track inspection [1], and a method for evaluating the internal state of health of embankments at the structure boundary. Therefore, we are developing a system that integrates various inspection data and ledger data from each field (called an 'integrated management system') [2]. This system enables us to browse and analyze data acquired in each field comprehensively, so that the running safety in addition to the quality of infrastructure management can be improved.

For integrated management of infrastructure data, it is important to unify the position information of the data. In Europe, the creation of an absolute position database for infrastructures is progressing (RINF project) [3][4]. This is because infrastructure manager and railway operator are different companies, and it is necessary to share position information of infrastructures between the companies. On the other hand, in Japan, one railway company generally manages infrastructure and operates railways, and the position information of infrastructure is often managed for each field.

At this time, we focus on a track management system (called LABOCS) introduced by railway companies in track maintenance work in Japan. LABOCS specializes in processing infrastructure ledger data and time-series data based on kilometerage and has functions that can analyze data multilaterally, and can manage high-density data such as track inspection data measured at intervals of $0.25 \mathrm{~m}$. However, LABOCS can handle only waveform and ledger data, and cannot handle data such as images, videos, and audio that are expected to be utilized by the integrated management system. Therefore, by utilizing LABOCS which has the above-mentioned characteristics, it is conceivable that the integrated management system can be efficiently developed by building on this system so that it can capture data from other fields and exploit data in other formats based on kilometerage.

This paper first summarizes the merits of integrated data management, and then describes the new functions we expect to build into the integrated management system. We also report the elemental technologies required to realize these functions and their development status. Furthermore, we show some examples of infrastructure maintenance using the integrated management system developed in this study.

\section{Present status of maintenance of railway infrastructure and necessity for integrated management}

In anticipation of aging infrastructure and a declining labor force, it is important to save labor for inspection, diagnosis, and maintenance of railway infrastructure maintenance. In the next example, we illustrate the need to improve railway infrastructure maintenance efficiency by factoring in data from different 'fields' by describing the state of progress in improving the efficiency of track maintenance cycles.

\subsection{State of progress in improving track maintenance cycle effi- ciency}

Track maintenance repeats the plan-do-check-act (PDCA) cycle of "inspection," "diagnosis," "planning," and "implementation" as shown in Fig. 1. Once the track condition is "inspected," a "diagnosis" is made to evaluate progress in deterioration of track geometry and of the track materials themselves. Accordingly, a maintenance is "planned" and "implemented" upon the plan. This study aims to develop and improve LABOCS and the track maintenance planning system [5] by supporting the process from "inspection" to "creation of a maintenance plan."

Currently, although LABOCS and the track maintenance planning system are being developed as individual systems, we are considering integrating them into one system. Specifically, LABOCS facilitates access to various inspection data by managing to integrate the data acquired from track inspections. In addition, LABOCS processes the data necessary for creating a maintenance plan, such as historical data of track inspection, and passes this processed data to the track maintenance planning system. Then, the planning system uses the processed data to perform prediction of track geometry deterioration and material condition diagnosis, and 


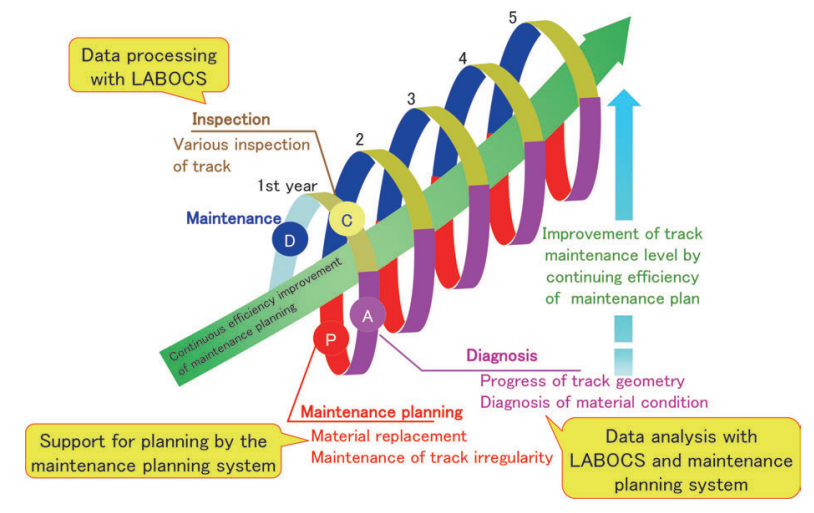

Fig. 1 Improving efficiency of the track maintenance

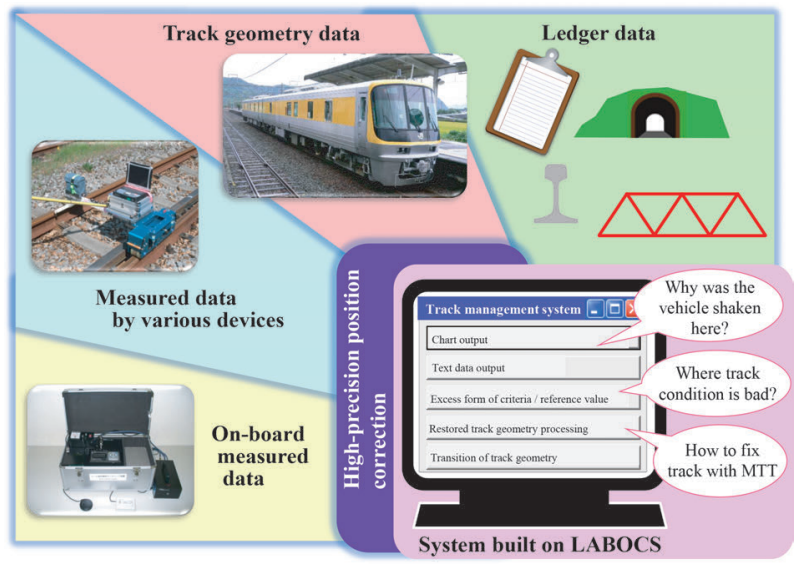

Fig. 2 Overview of LABOCS

outputs a track geometry maintenance plan and a material replacement plan. We carry out maintenance based on the outputs from the above flow, and proceed to the next cycle starting from inspection. The aim of this is to develop the LABOCS and the integrated management system, so that they can support continuous improvement in efficiency, such as for track maintenance cycles.

\subsection{Overview of LABOCS and need for integrated management of data from other fields}

As shown in Fig. 2, LABOCS is software that can analyze and process various data for track maintenance, such as track geometry, vehicle vibration, and infrastructure ledger data multilaterally [6].

Many manufacturers and railway companies have developed equipment for track inspection and monitoring, and Railway Technical Research Institute has also developed and put into practical use some equipment [7][8]. Conventionally, the data obtained from these devices are generally managed separately by the dedicated software or ledger attached to each device, and used to judge the necessity of maintenance of individual infrastructure by comparing with certain criteria. In addition, for each inspection item, the relation between the items can often only be analyzed by printing and comparing inspection results individually. Therefore, in order to cross-check and analyze inspection data comprehensively, to diagnose failure events and investigate causes, and to examine appropriate maintenance methods, it is desirable to be able to manage individual data collectively and easily analyze the relationship between these data. This issue is not limited to track data, therefore it is conceivable that the same analysis, investigation of cause, and examination of maintenance methods through integrated data management, may be applicable in other fields.

Given the broad potential of integrating data management, we
Table 1 Examples of needs for integrated management of data in other fields

\begin{tabular}{|c|c|c|}
\hline Utilization field & Utilization data & Utilization example \\
\hline \multirow{3}{*}{$\begin{array}{c}\text { Needs for structure } \\
\text { management utilizing track } \\
\text { data }\end{array}$} & \multirow{3}{*}{$\begin{array}{l}\text { Information of track } \\
\text { maintenance (especially } \\
\text { longitudinal level irregularity) }\end{array}$} & $\begin{array}{l}\text { Time-course change in the } \\
\text { camber of the bridge }\end{array}$ \\
\hline & & $\begin{array}{l}\text { Evaluation of settlement in } \\
\text { embankment sections }\end{array}$ \\
\hline & & $\begin{array}{l}\text { Detection of various } \\
\text { deformations }\end{array}$ \\
\hline \multirow[b]{2}{*}{$\begin{array}{c}\text { Needs for track management } \\
\text { utilizing structural data }\end{array}$} & $\begin{array}{l}\text { Information on clearance gauge } \\
\text { such as stations and tunnels }\end{array}$ & $\begin{array}{l}\text { Improving inspection } \\
\text { efficiency }\end{array}$ \\
\hline & $\begin{array}{l}\text { Inspection data in the } \\
\text { embankment section }\end{array}$ & $\begin{array}{l}\text { Detection of deformation by } \\
\text { comparison with the } \\
\text { condition and transition of } \\
\text { track geometry }\end{array}$ \\
\hline
\end{tabular}

investigated and organized current needs in different fields, which could be met by integrated data management. As an example, Table 1 shows the needs identified in track and civil engineering maintenance management. The table indicates that, in both fields, there is a need to utilize data from other fields to detect deformations in infrastructure. Similar needs exist not only between track and civil engineering but also between other fields. Therefore, when developing LABOCS into the integrated management system, it is important to be able to manage data from other fields such as civil engineering in addition to the conventional track related data. As a result, it will be possible to improve the quality of infrastructure management. However, similar to tracks, there are also various inspection devices in other fields that have not been dealt with so far. Therefore, it is an issue to determine the specifications of the integrated management system after thoroughly investigating what kind of data is handled.

\subsection{New functions needed with integrated management}

We examined the functions that the integrated management system should have in order to meet the needs for the system shown in the previous section.

For example, Table 2 shows the results of organizing the needs for new functions relating to tracks. From the table, there are many needs for utilization of position information of Global Navigation Satellite System (GNSS) and real-time information sharing. Among the needs, the functions of automatically converting position information of GNSS to kilometerage, pasting photos and voice memos to track geometry charts, and sharing data between groups are important functions that can be used in common in all fields. It is noted that since the current LABOCS specializes in processing based on kilometerage as position information, it is not possible to handle data that does not have kilometerage such as photos and videos. Accordingly, it is necessary to develop a method for uniformly assigning position information to data that does not have kilometerage. There is also a need for remote meetings, as shown in the table. Currently, it is generally used to confirm the acquired data at the office and utilize it for examining maintenance methods and creating maintenance plans. If the office and the site can be connected by communication link, for example, by immediately sharing photos and videos acquired at the site, it will be possible to immediately determine the need for maintenance through remote meetings. Furthermore, if the situation is urgent, it will be possible to take flexible measures such as giving instructions for first aid on the spot.

\subsection{Merits of integrated management and system development}

In sum, by managing a variety of integrated data, the integrated management system developed in this study can be used for detecting infrastructure deformation, investigating the cause of defects, and examining appropriate maintenance methods. Considering that the maintenance cycle shown in Fig. 1 can be applied to railway infrastructure in general, integrated management has merits in terms of "diagnosis" and "creation of maintenance plans." 


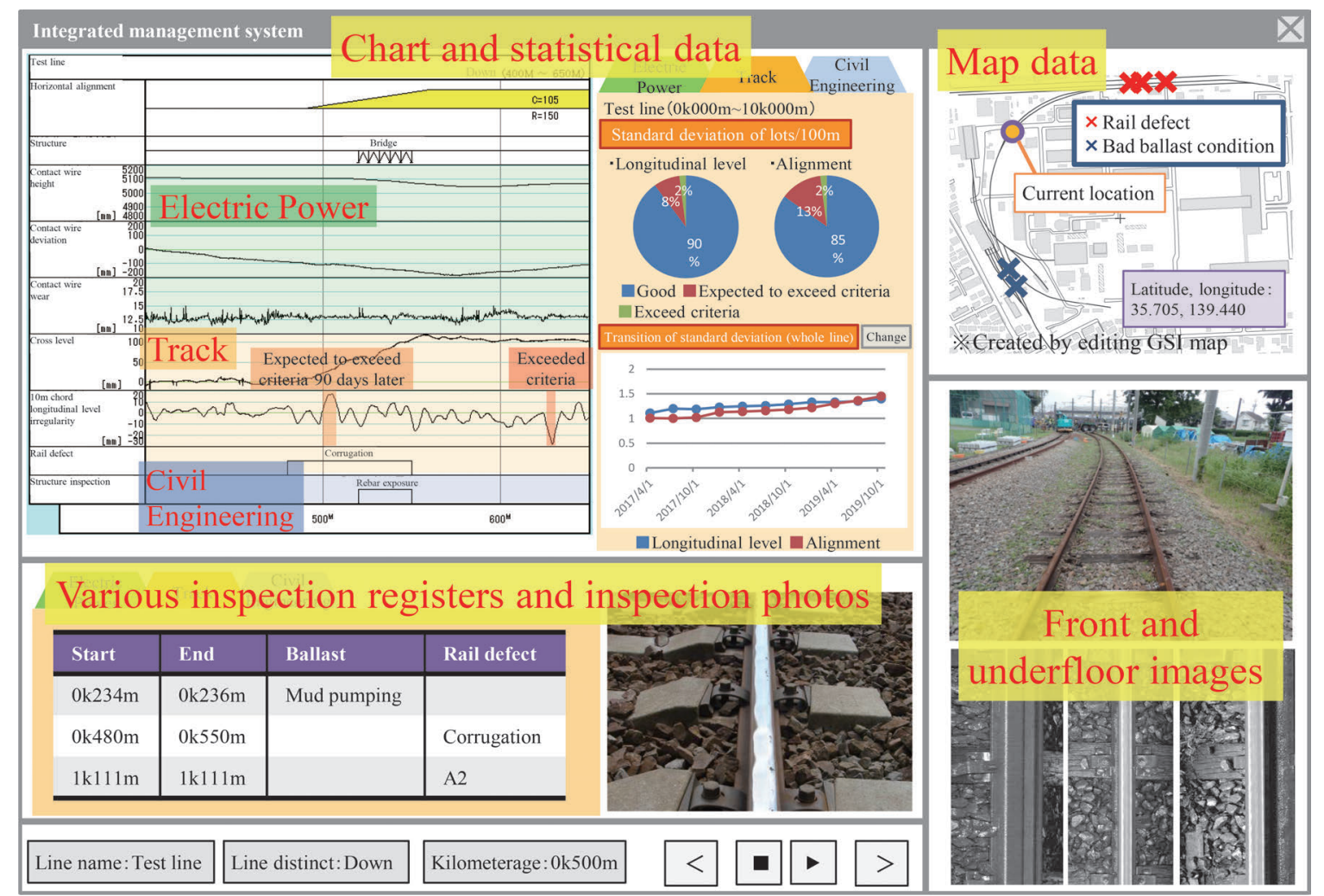

Fig. 3 Main screen image of the completed integrated management system

Table 2 Examples of needs for integrated management of data in other fields

\begin{tabular}{|c|c|c|}
\hline Classification & Proposal function & Purpose \\
\hline \multirow{3}{*}{$\begin{array}{l}\text { Utilization of } \\
\text { position } \\
\text { information of } \\
\text { GNSS }\end{array}$} & $\begin{array}{l}\text { Automatic display of track geometry chart on-site } \\
\text { linked with position information of GNSS }\end{array}$ & Streamlining of track patrol \\
\hline & $\begin{array}{l}\text { A function that automatically converts GNSS data } \\
\text { of photos taken at the site to kilometerage linked } \\
\text { with track geometry }\end{array}$ & Streamlining of track patrol \\
\hline & $\begin{array}{l}\text { A navigation system that presents access routes to } \\
\text { entry points into railway track and displays } \\
\text { parking areas }\end{array}$ & $\begin{array}{l}\text { Correspondence to mobile } \\
\text { organization of track maintenance }\end{array}$ \\
\hline \multirow{4}{*}{$\begin{array}{l}\text { Real-time } \\
\text { information } \\
\text { sharing }\end{array}$} & $\begin{array}{l}\text { A function to automatically create a recommended } \\
\text { MTT plan from the historical track geometry of } \\
\text { any section and share it among users }\end{array}$ & $\begin{array}{l}\text { Simplification and remoteization } \\
\text { of construction review meetings }\end{array}$ \\
\hline & $\begin{array}{l}\text { Integration of on-site VR data and track inspection } \\
\text { data obtained by the automatic patrol system }\end{array}$ & $\begin{array}{l}\text { Simplification and remoteization } \\
\text { of construction review meetings }\end{array}$ \\
\hline & Introducing a teleconference system & $\begin{array}{l}\text { Remoteization of disaster / } \\
\text { accident countermeasure meetings }\end{array}$ \\
\hline & $\begin{array}{l}\text { A function of pasting handwritten memos, photos } \\
\text { and voice memos to track geometry chart, and } \\
\text { sharing of these additional data between groups }\end{array}$ & Remote group work \\
\hline
\end{tabular}

For users to benefit from the above-mentioned merits of integrated management, it is important for the integrated management system to be able to uniformly handle different types of data from each field. In addition, since the types of data are diverse corresponding to inspection registers and photos managed in each field, it is important to be able to comprehensively browse and analyze these data.

\section{Integrated management system}

Figure 3 is an image of the main screen of the completed integrated management system under development. Up to now, we have developed a prototype of the system that implements an interface that can simultaneously display charts which have been realized with conventional LABOCS, inspection registers linked by convert- ing position information to kilometerage, inspection photos, map, and videos. In the following, we explain the functions that are expected to be implemented to complete the integrated management system, including the developed functions.

\subsection{Main functions implemented in the integrated management system}

\subsubsection{Display of chart of inspection data and statistical data}

With the current LABOCS, it is possible to display a chart of time-series data centered on the specified location by inputting the line name, line distinct, and kilometerage. As display items, track inspection data, vehicle vibration data, and various inspection registers can be displayed. It is also possible to calculate statistical data 
for time-series data. For example, Fig. 3 shows a frequency distribution of standard deviation of lots/100 m of track geometry and a transition diagram of standard deviation of the whole line section, along with a chart of each management item. It is possible to switch between whole line display and lot display for the transition diagram of standard deviation. Furthermore, for example, as highlighted in red or orange on the left side of the $10 \mathrm{~m}$-chord longitudinal level irregularity in the figure, information on where the track geometry exceeds or is expected to exceed the criteria is output on the chart. At this time, current LABOCS can be used to output the locations where the track geometry exceeds the criteria as of the inspection date. On the other hand, the trend analysis results of track geometry are reflected in the output of expected criteria excess location by utilizing the historical data used in the maintenance planning system. As a result, in addition to being able to manage the average status of the whole section, by checking the transition for each lot and the locations where the track geometry exceeds the criteria, it can be used to examine the locations that require maintenance and the maintenance implement timing.

The integrated management system implements the above functions for data in other fields as well. For example, in Fig. 3, a chart of contact wire wear inspection data is displayed as overhead contact line related data. Similar to the above display example in the track field, we can consider maintenance location and maintenance timing of contact wire by displaying the transition of the amount of wear.

\subsubsection{Display of various inspection registers and inspection photos}

Conventionally, inspection registers were generally managed separately for each inspection type. On the other hand, the integrated management system displays the results of various inspections together with kilometerage in the same list. Then, when we click any row on the list, the chart screen moves to the vicinity of the kilometerage, and at the same time, if an inspection photo is registered, the photo is also displayed. In this way, by viewing the results of individual inspections, charts, and photos on the same screen, it is possible to easily confirm the track condition of defective points. As a result, for example, by simultaneously viewing current track geometry and transition of track geometry of the section where corrugation exists as shown in Fig. 3, not only we can grasp the degree of corrugation with photos, we can also confirm the effect of corrugation on track geometry deterioration. If it is confirmed that the influence of corrugation is large, it can be used for considering combination maintenance of rail grinding and tamping with a multiple tie tamper (MTT) [9] to suppress track geometry deterioration.

\subsubsection{Display of map data}

By linking chart and various inspection data with map, in addition to displaying a map centered on a specified kilometerage, a geographic information system (GIS) function that visualizes locations that require maintenance will be implemented. By displaying the information of defective points registered in the inspection register on the map for each inspection type, it is possible to easily recognize the spots requiring maintenance on the map. For example, since there is a section where rail defects are concentrated on the map in Fig. 3, we can recognize the area where rail defects exist and choose the area to replace rail referring to the list in Section 3.1.2.

\subsubsection{Display of front and underfloor images}

The system imports video taken by a frontal camera linked to kilometerage of the chart and displays the photo of the specified kilometerage. This makes it possible to confirm the track condition and environment along the specified kilometerage. In recent years, it has become common to utilize videos taken by frontal cameras on commercial trains. The system incorporates clearance disorder, ob- jects near the clearance and changes in the environment along the railway line output by the system, into the integrated management system. Then, we can confirm the detected abnormalities such as the inclination of the pillars and building plants on the system while referring to the ledger, and can promptly consider the countermeasures. In addition, by confirming the video in conjunction with the chart, it is possible to confirm the surrounding condition with the video at the same time as the track geometry at the location where the large vehicle vibration occurred.

\subsection{Elemental technologies and states of development}

In order to realize the functions implemented in the integrated management system shown above, it is necessary to develop some elemental technologies. In the following, we show such elemental technologies along with the progress of their development.

\subsubsection{Unification of position information}

As mentioned above, there is data that does not have kilometerage as position information. In addition, there are "construction kilometerage" and "management kilometerage" in kilometerage, and the position pointed to by the same kilometerage may differ depending on the field and inspection data. Therefore, to manage to integrate these data, it is necessary to unify the position information. Since the integrated management system is based on LABOCS, we unify to the kilometerage handled in track field.

The integrated management system is equipped with a "kilometerage-latitude/longitude control master" as shown in Fig. 4, so that the position information is unified by referring to this master. In order to compare the kilometerage with latitude/longitude, the prototype prepares a detailed comparison table as the master as shown in Table 3 for each kilometerage in advance. However, this method has a problem that it is necessary to manually assign the control information of kilometerage-latitude/longitude for each newly acquired data, which requires a large amount labor. Therefore, we have developed a method to automatically assign kilometerage to data with latitude/longitude information [7]. Figure 5 shows the principle underlying the developed method in which the kilometerage is assigned from the positional relationship of three points using the latitude/longitude information of the kilometer posts that are the first and second closest to the point to be assigned kilometerage. Figure 6 is an image of the automation of kilometrage assignment by applying this method. With this method, it is possible to automatically assign kilometerage to new additional data with latitude/longitude by creating a list of latitude/longitude of kilometer posts as shown in Table 4.

\subsubsection{Synchronization of video data and chart position}

Since video data does not generally have position information, it is necessary to assign kilometerage to the video data so that it can

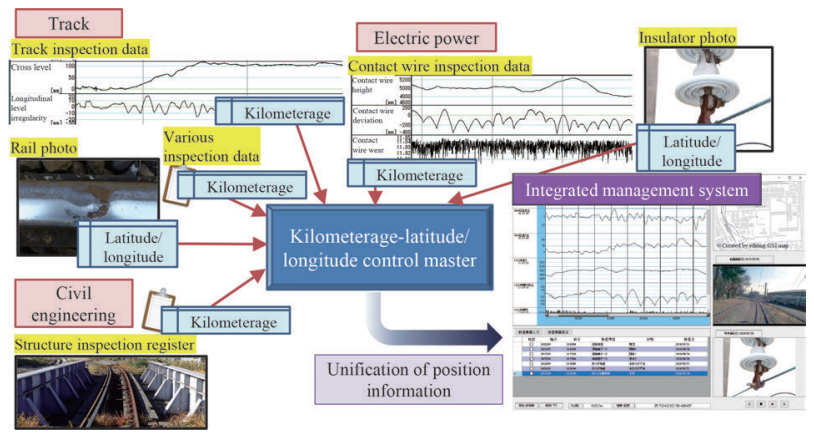

Fig. 4 Unified location information in the integrated management system 


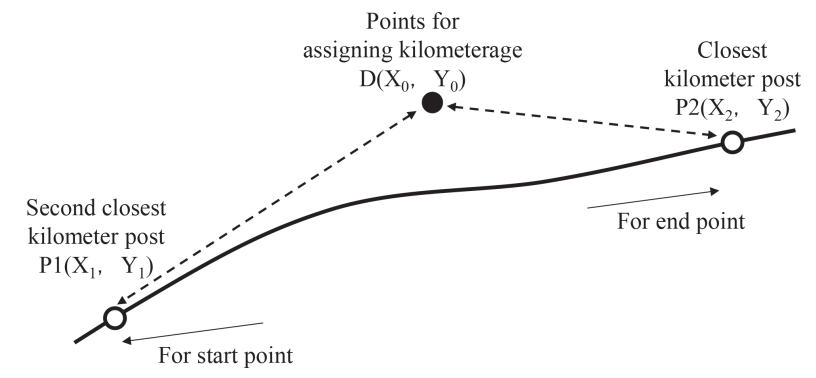

Fig. 5 Image showing how kilometerage to point $D$ is assigned with latitude/longitude system

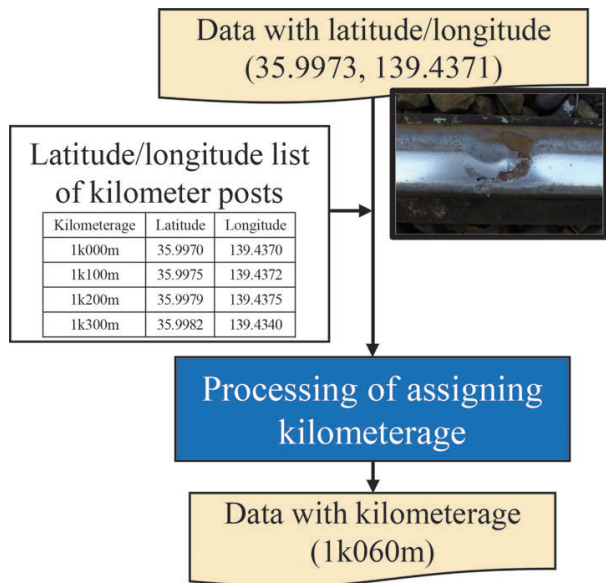

Fig. 6 Image of automation for assigning kilometerage

be handled by the integrated management system. If it becomes possible to assign kilometerage to the video data, index values such as track geometry and vehicle vibration of the current position can be displayed on the moving image, and the condition of the infrastructure can be confirmed while watching the video.

Figure 7 shows a display image of the statistical data of the track geometry on the video. Similarly, any distance-sampled data can be displayed on the video.

\section{Examples of how the integrated management system can be used}

In this chapter, we show examples of infrastructure maintenance using the integrated management system.

4.1 An example of creating a rail maintenance plan by considering track condition

Railway companies create track maintenance plans such as for tamping by MTT, ballast replacement, and rail replacement based on the results of various track inspections. Currently, it is necessary to comprehensively evaluate the inspection results of multiple items, and consider an appropriate maintenance method and maintenance timing.

Figure 8 shows an example of displaying track inspection data and a rail defects register in the prototype of the integrated management system. In this figure, the longitudinal level irregularity and axle-box acceleration measured by the track inspection car, the inspection result of the rail defect detect car, and the photo of the rail defect are displayed at the same time. In this section, a rail defect already exists, and as a result, the vertical axle-box acceleration increases. In such locations, rail grinding cannot remove the rail de-

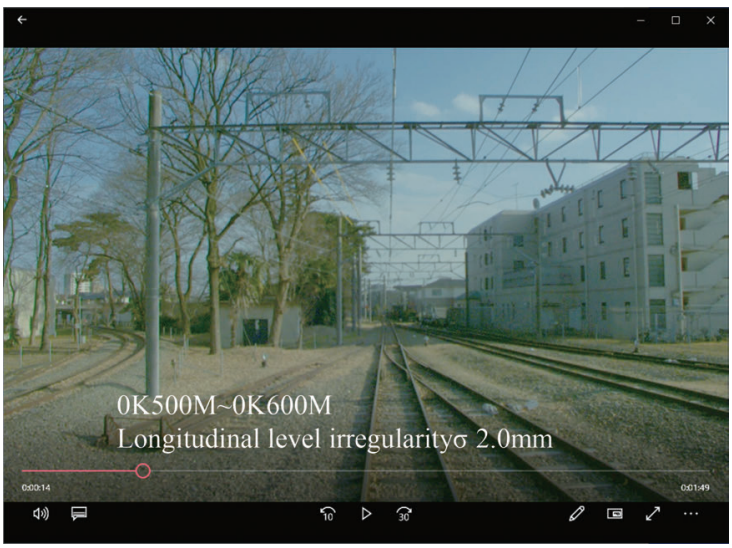

Fig. 7 Image of statistical data displayed on the video

Table 3 An example of kilometerage-latitude/longitude comparison table

\begin{tabular}{|c|c|c|}
\hline Kilometerage & Latitude & Longitude \\
\hline $0 \mathrm{k} 400 \mathrm{~m}$ & 35.70133 & 139.44 \\
\hline $0 \mathrm{k} 401 \mathrm{~m}$ & 35.70134 & 139.44 \\
\hline $0 \mathrm{k} 402 \mathrm{~m}$ & 35.70135 & 139.44 \\
\hline $0 \mathrm{k} 403 \mathrm{~m}$ & 35.70136 & 139.44 \\
\hline
\end{tabular}

Table 4 An example of latitude/longitude list of kilometer posts

\begin{tabular}{|c|c|c|}
\hline Kilometerage & Latitude & Longitude \\
\hline $1 \mathrm{k} 000 \mathrm{~m}$ & 35.9970 & 139.4370 \\
\hline $1 \mathrm{k} 100 \mathrm{~m}$ & 35.9975 & 139.4372 \\
\hline $1 \mathrm{k} 200 \mathrm{~m}$ & 35.9979 & 139.4375 \\
\hline $1 \mathrm{k} 300 \mathrm{~m}$ & 35.9982 & 139.4340 \\
\hline
\end{tabular}

fect. Therefore, this should lead to a decision to replace the rail. In this way, by unifying the data managed individually, it becomes possible to select the appropriate type of maintenance.

4.2 Examples of status diagnosis and creating a maintenance plan using data from multiple fields

\subsubsection{An example of resonant bridge extraction using track} data

The method for extracting resonant bridges using track inspection data from onboard measurements mentioned at the beginning of this paper, is an example of how infrastructure diagnoses can be improved by using data from different 'fields' [1]. Conventionally, detecting a resonant bridge from the ground, requires costly inspections to measure deflection in each bridge when vehicles pass over it. On the other hand, this method performs waveform processing that emphasizes the vibration component peculiar to the resonant bridge with respect to the longitudinal level irregularity or vertical car-body acceleration measured in the head and tail train set. After that, the resonant bridge is extracted by performing the difference processing between the head and tail train set. Therefore, this method can significantly reduce the labor required for onsite in-person measurements.

This method of extracting resonant bridges has already been implemented in the current LABOCS and can be used as a function 
for structure management in the integrated management system.

\subsubsection{An example of creating a tamping plan with MTT using track and electric power data}

When creating a tamping plan with MTT for tracks, it is necessary to determine how much tracks need to be raised and moved track in the horizontal direction so as not to exceed the criteria of the contact wire height and contact wire deviation of overhead contact line managed through the 'field of' electric power. Conventionally, when planning such construction, it is necessary to have a preliminary meeting between the electric power group and track group. On the other hand, in the integrated management system, both the track geometry and contact wire height and deviation measured by the inspection car can confirmed on the same chart. Therefore, it is possible to decide the upper limit of the amount of track raising at each location without conducting preliminary meeting, in order reduce required for creating a tamping plan with MTT.

\section{Conclusions}

In this paper, we organized the merits of integrated management of various data for railway infrastructure maintenance, and showed some functions to be implemented in the integrated management system. We also reported the elemental technologies required for these functions and their development status. In addition, we showed an example of infrastructure maintenance using the integrated management system.

In the future, we studied methods to adopt various data into the integrated management system, such as the data specifications of inspection equipment handled in each field. In addition, we focus on applying the technologies that are being developed individually, such as the kilometerage assigning method and video utilization technologies, to the integrated management system.

\section{References}

[1] Matsuoka, K., Watanabe, T. and Uehan, F., "Resonant Bridge Detection Method by On-board Measurement," QR of RTRI, Vol. 61, No. 2, pp. 133-138, 2020.

[2] Yoshida, T., Tanaka, H., Nishimoto, M. and Miwa, M., "Examination of method for assigning unified kilometerage to railway infrastructure management data and deployment to unified management system," J-RAIL2020, S2-2-6, 2020 (in Japanese).

[3] Commission Implementing Decision 2011/633/EU of 15 September 2011 on the common specifications of the register of

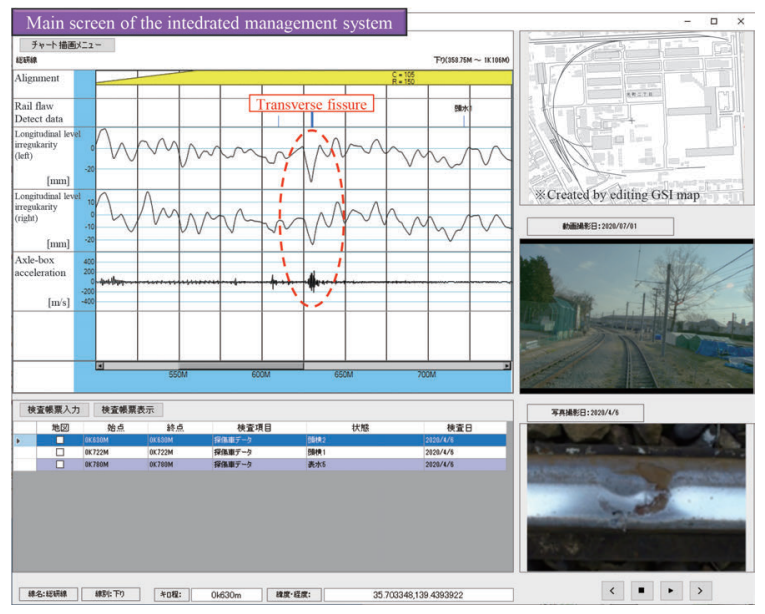

Fig. 8 An example of merging track inspection data and rail inspection data using the prototype integrated management system

railway infrastructure.

[4] European Union Agency for Railways, "RINF - Register of Insrastructure," https://rinf.era.europa.eu/RINF/, 2022.01.06 access.

[5] Miwa, M., Yamamoto, S., Matsumoto, M., Oshima, T. and Yoshida, Y., "Modeling a Track Condition Prediction and Optimizing Mid-term and Long-term Track Maintenance Plan," RTRI Report, Vol. 33, No. 2, pp. 11-16, 2019 (in Japanese).

[6] Yoshimura A., Yoshida Y., Hosokawa T. and Kamiyama M., "Development of Database System, Micro LABOCS-II+, for Railway Track Maintenance Management," $Q R$ of RTRI, Vol. 36, No. 2, pp. 95-101, 1995.

[7] Tanaka, H. and Shimizu, A., "Practical Application of Rail Surface Roughness Continuous Measurement Trolley for Maintenance of Rail Corrugation," QR of RTRI, Vol. 57, No. 2, pp. 118-124, 2016.

[8] Nakamura, T., Momoya, Y., Tanigawa, H., Kikkawa, S. and Kijiya, I., "Development on the Evaluation Method of Track Support Stiffness of the Ballasted Track," RTRI Report, Vol. 31, No. 12, pp. 29-34, 2017 (in Japanese).

[9] Matsumoto, M., Miwa, M., Yoshida,T., Yasaka, K., Oketani,E. and Harada, Y., "Modeling an Optimal Track Maintenance Schedule in Consideration of the Timing of Grinding and Tamping," QR of RTRI, Vol. 58, No. 3, pp. 229-235, 2017.
Authors

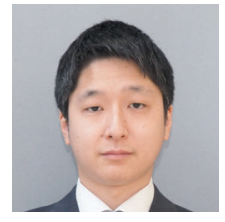

Takafumi YOSHIDA

Assistant Senior Researcher, Track Geometry and Maintenance Laboratory, Track

Technology Division

Research Areas: Track Geometry

Management, Maintenance Support System

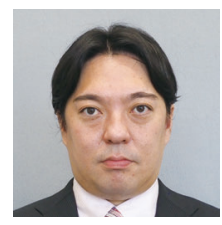

Hirofumi TANAKA, Dr. Eng.

Senior Researcher, Track Geometry and

Maintenance Laboratory, Track Technology

Division

Research Areas: Track Geometry

Management, Signal Processing of Track

Geometry Data, On-board Monitoring

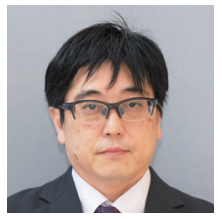

Masahito NISHIMOTO

Senior Researcher, Track Geometry and Maintenance Laboratory, Track Technology Division

Research Areas: Track Geometry

Management

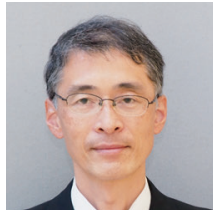

Masashi MIWA, Ph.D.

Director, Head of Track Technology Division Research Areas: Track Geometry Management, Maintenance Support System 